\title{
Inteligencias multiples y rendimiento academico en estudiantes de instituciones educativas privadas del sector urbano de trujillo 2014
}

\author{
Multiple intelligences and academic performance in students of private educational \\ institutions of the urban sector of trujillo 2014 \\ Inteligências multiplas e rendimento acadêmico em estudantes de instituições \\ educativas privadas do setor urbano de trujillo 2014
}

DOI: http://dx.doi.org/10.21803\%2Fpenamer.11.21.529

\section{Jorge Arturo Cavero Carranza}

Emerita Victoria Vásquez Tolentino https://orcid.org/0000-0002-4401-7977

\section{¿Cómo citar este artículo?}

Cavero, J. \& Vásquez, E. (2018). Inteligencias multiples y rendimiento academico en estudiantes de instituciones educativas privadas del sector urbano de trujillo 2014. Pensamiento Americano, $71(21), 127-135$

DOI:http://dx.doi.org/10.21803\%2Fpenamer.11.21.529

\section{Resumen}

La teoría de las inteligencias múltiples es un tema innovador en el área de la Psicología Educativa, ya que permite al estudiante y educador identificar con facilidad cuales son las áreas académicas fuertes y débiles. La finalidad del estudio fue encontrar la correlación entre inteligencias múltiples y el rendimiento académico, teniendo como muestra a 115 estudiantes de tres Instituciones Educativas. Estudio descriptivo correlacional. Se aplicó la prueba de inteligencias múltiples, también se recopilaron las notas promedio por asignatura. Para encontrar la correlación entre las variables se usó el estadístico rho de Spearman, encontrando que el rendimiento académico es el previsto para el grado académico, las inteligencias interpersonal, espacial y naturalista son las más desarrolladas entre los estudiantes, la inteligencia lingüística y matemática son la de menor desarrollo, no hubo diferencia del logro en el rendimiento académico y las inteligencias múltiples según el sexo de los estudiantes. Ante estos resultados se determinó que a mayor desarrollo de las inteligencias múltiples se tendrá un mejor rendimiento académico.

PALABRAS CLAVE: Inteligencias Múltiples, Rendimiento Académico.

\begin{abstract}
The theory of multiple intelligences is an innovative topic in the area of Educational Psychology, since it allows the student and educator to easily identify which are the strong and weak academic areas.

The purpose of the study was to find the correlation between multiple intelligences and the academic performance, taking as a sample 115 students from three Educational Institutions. The correlational descriptive study was applied the multiple intelligences test, also the average grades per subject were compile.

To find the correlation between the variables was used the Spearman rho statistic, finding that the academic performance is the one expected for the academic degree, the intelligences interpersonal, spatial and naturalistic are the most developed among the students, the linguistic and mathematical intelligence was of minor development, and there was no difference of achievement in academic performance and multiple intelligences according to the sex of the students.

Given these results, it was determined that the higher the development of multiple intelligences, will be achieved the better academic achievement.
\end{abstract}




\title{
Resumo
}

\begin{abstract}
A teoria das inteligências múltiplas é um tema inovador na área da Psicologia Educativa, já que permite ao estudante e educador identificar com facilidade quais são as áreas acadêmicas fortes e débeis. A finalidade do estudo foi encontrar a correlação entre inteligências múltiplas e o rendimento acadêmico, tendo como amostra a 115 estudantes de três instituições educativas. Estudo descritivo correlacional. Se aplicou o teste de inteligências múltiplas, também se compilaram as notas promedio por assinatura. Para encontrar a correlação entre as variáveis se usou o estatístico rho de Spearman, encontrando que o rendimento acadêmico é o previsto para o grau acadêmico e as inteligências múltiplas segundo o sexo dos estudantes. Frente a estes resultados, se determinou que ao maior desenvolvimento das inteligências múltiplas se terá um melhor rendimento acadêmico.
\end{abstract}

PALAVRAS-CHAVE: Inteligências Múltiplas, Rendimento Acadêmico.

\section{Perfil}

Docente Universidad San Pedro, Estadístico- Ministerio del Interior 128
Jorge Arturo Cavero Carranza

Ingeniero Estadístico

Emerita Victoria Vásquez Tolentino

Licenciada en psicología

Dra. en Gestión y Ciencias de la Educación

Docente Nombrado: Universidad San Pedro - Perú. tvve07@gmail.com 


\section{Introducción}

E n Guatemala, Pérez S.J. (2015) realizó una investigación cuyo tema fue relacionado a las Inteligencias Múltiples más desarrolladas en estudiantes con bajo rendimiento en Matemática, en el municipio de San José Pínula; cuya finalidad fue robustecer el desarrollo de la enseñanza y aprendizaje para mejorar el desempeño laboral del docente, al identificar las inteligencias múltiples con mayor desarroIlo entre los estudiantes con bajo nivel en Matemática. La investigación fue cuantitativa, no experimental, se aplicó a 32 estudiantes el test de inteligencias múltiples. Entre los resultado, el autor determinó que se tiene más a estudiantes que no tienen desarrolladas de forma equilibrada las inteligencias múltiples y que presentan predominantemente las inteligencias de tipo musical/rítmica. La inteligencia interpersonal está desarrollada en el $56.25 \%$ de los estudiantes, la intrapersonal en $56.25 \%$, Musical 59.38\%, Kinestésico 15.63\%, Espacial 6.25\%, Matemática $15.63 \%$ y verbal $25 \%$. Concluyendo el autor, que casi la totalidad de los estudiantes que presentaron bajas puntuaciones en el área de matemática, no presentan un buen nivel en la inteligencia lógico-matemática.

Asimismo, Aliaga, Ponce, Bulnes, Elizarde, Montgomery, Gutierrez, Delgado, Perea, y Torchiani (2012). Desarrollo un estudio referente a las inteligencias múltiples, acerca de la identificación y relación con el rendimiento en el área de matemática entre los estudiantes del último año de educación secundaria en la ciudad de Lima. Cuyo objetivo final de estudio fue encontrar la relación entre las inteligencias múltiples y el logro del rendimiento académico en el área de matemáticas. Con una metodología descriptiva-correlacional, en este estudio participaron 14 centros educativos entre estatales y privados de Lima. Encontrando entre sus resultados que la inteligencia lógico - matemática presenta mayor correlación con el rendimiento en el área de matemáticas $(\mathrm{p}=0.000)$ que con la puntuación promedio de las notas ( $p=0.015)$. Asimismo, determinaron también que la inteligencia lingüística está correlacionada con la puntuación promedio de las notas ( $p=0.005)$ y con la puntuación obtenida en matemáticas; por otro lado la inteligencias musical, corporal-cinestésica, intrapersonal y espacial, tienen una menor importancia, por lo cual concluyeron que las inteligencias múltiples si se relacionan significativamente (Higuera, 2013).

Las inteligencias múltiples, es una teoría de visión moderna en el área de la educación, ya que su identificación permite a maestros y estudiantes, identificar mejor sus puntos intelectuales de mayor y menor fortaleza; y de esta manera, estimular aquellas inteligencias menos desarrolladas. Por ser un tema de actualidad, es necesario desarrollar investigaciones en el nivel básico de educación primaria, en tanto que es una edad que permite una mejor estimulación y desarrollo de las inteligencias múltiples, por consiguiente, se concretizó el trabajo de investigación en un grupo de estudiantes del nivel primario que requiere de un conocimiento fundamentado de su realidad educativa y esto puede conseguirse realizando trabajos de investigación por parte de los docentes de las diferentes instituciones privadas del sector urbano y en general de Trujillo (Fernández, 2016) . Por ello nos planteamos como problema de investigación: ¿Cuál es la correlación entre las inteligencias múltiples y el rendimiento académico en estudiantes de las Instituciones Educativas Privadas del sector urbano de Trujillo 2014?

\section{Inteligencias Múltiples}

Para Flores (2012) la inteligencia evoluciona y mejora por la interacción entre las características genéticas del individuo y el medio ambiente que los rodea, es decir que, resulta de la influencia recíproca entre las peculiaridades heredadas y la coyuntura ofrecida por el ambiente donde vive el individuo. Lo cual 
concuerda con lo que se refiere en la Teoría de las Inteligencias Múltiples propuesto por Gardner (1983), quien definió la inteligencia como "la capacidad para resolver problemas y crear productos valorados, al menos en un contexto cultural o en una comunidad determinada", en esta línea manifiesta, que, a pesar de identificar diferentes tipos de inteligencia jamás existirá una aislada de otra o de manera independiente, sino que lo hacen de manera agrupada y complementaria. La inteligencia no es una sola, sino que es la reunión de diferentes inteligencias entre ellas tenemos: musical, matemática, lingüística, cinestésica, espacial, intrapersonal, interpersonal y natural, En la actualidad se está estudiando la inteligencia espiritual o existencial (Gardner, 1983).

Inteligencia musical: Es la habilidad para sentir, diferenciar, convertir y manifestar los diferentes sonidos y formas musicales. Esta inteligencia está relacionada con el canto, interpretar un instrumento y apreciar una pieza musical. (Flores M., 2010).

Inteligencia lógico-matemática: Está relacionada con la aptitud numérica, es decir trabajar y entender cuestiones numéricas, deducción de evidencias, resolver preguntas lógicas, y en su mayoría del pensamiento científico, ayuda a resolver problemas matemáticos enunciados oralmente y por escrito. (Gardner, 2011).

Inteligencia Lingüística. Es el talento para usar correcta y eficazmente un lenguaje, de manera verbal o escrita, con adecuado uso de la semántica, de pronunciación y del uso práctico del lenguaje. (Castillo, 2013).

Inteligencia corporal- kinestésica: Es la habilidad en la expresión corporal y el adecuado uso de objetos, expresándose en diversas actividades, compromete la capacidad para emplear el propio cuerpo en su totalidad o diferentes áreas del mismo (Barraza \& Gonzales, 2016).
Inteligencia espacial: Es el don para sentir el mundo visual, entender y manejar los espacios, ejecutar modificaciones a las percepciones iniciales, y rehacer características de la experiencia visual, así estemos sin los estímulos físicos adecuados (Gardner, 2011).

Inteligencia intrapersonal: Está asociada con la habilidad para entenderse a sí mismo, poder interactuar con sus emociones y sentimientos propios de si, con la finalidad de auto comprenderse y dirigir su propia vida (Gardner, 2011).

Inteligencia interpersonal: Ayuda a entender las pretensiones, motivaciones, estados de ánimo y personalidades de otros individuos, se manifiesta a través de aspectos lingüísticos y no lingüísticos (Gardner, 2011). Inteligencia naturalista: Esta inteligencia aporta en la atracción y comprensión del medio ambiente que nos rodea, es la habilidad de reconocer el lenguaje natural y fascinación ante el paisaje natural o no. (Antunes, 2005).

\section{Rendimiento Académico}

Es el nivel de logro en el aprendizaje, que alcanza el alumno en el ámbito académico. Se logra medir mediante una evaluación académica, comprendidas como el conglomerado de métodos que se programan y suministran dentro del desarrollo educativo, con la finalidad de conseguir información del alumno para valorar su logro académico. Para poder medirlo, el MINEDU (2016) estableció niveles en base a los logros alcanzados por el estudiante, se establecen 4 dimensiones

AD (Logro destacado) El educando demuestra el logro de los aprendizajes previstos, manifestando un manejo adecuado y satisfactorio de las tareas

A (Logro previsto) El educando demuestra haber logrado los aprendizajes previstos.

B (En proceso) El estudiante está en curso de alcanzar los aprendizajes previstos, para ello 
necesita apoyo académico durante un periodo razonable para lograrlo.

C (En inicio) El estudiante está comenzando a mostrar los aprendizajes previstos o evidencia inconvenientes para el manejo de éstos, requiriendo un mayor periodo de apoyo e intromisión del docente acorde con su ritmo y estilo de aprendizaje.

Tabla 1.

Evaluación de los aprendizajes en el nivel Primaria

\begin{tabular}{l|l|l}
\hline Escala & Dimensión & Nota \\
\hline AD & Logro destacado & $8-20$ \\
\hline A & Logro previsto & $14-17$ \\
B & En proceso & $11-13$ \\
C & En inicio & $0-10$ \\
\hline
\end{tabular}

\section{Hipótesis}

Existe correlación entre las inteligencias múltiples y el Rendimiento académico en estudiantes de Instituciones Educativas Privadas del sector urbano de Trujillo 2014

\section{Objetivos de la investigación}

Determinar la relación entre las inteligencias múltiples y el Rendimiento académico en estudiantes de Instituciones Educativas Privadas del sector urbano de Trujillo 2014.

\section{Material y metodos}

Se midió y evaluó diferentes dimensiones y tuvo como finalidad identificar la relación entre las variables pertinentes, según Hernández Fernández y Baptista (2014) este tipo de estudios es del tipo descriptivo-correlacional. Así mismo la población fue de 165 alumnos del $2^{\circ}, 3^{\circ}$ y $4^{\circ}$ grado de primaria, de tres Instituciones Educativas en Trujillo matriculados en el año académico 2014. Se escogió una muestra $n$ del total de la población, de este modo cada muestra posible de tamaño $\mathrm{n}$ tiene la misma probabilidad de ser escogida, este muestreo es llamado muestreo aleatorio simple (Scheaffer R.,
Mendenhall W. \& Ott L. 2007). La muestra final quedo con 115 alumnos.

Técnicas e Instrumentos De Investigación: Se empleó el test psicométrico y el análisis documental como técnicas de recolección, (Hernández Fernández \& Baptista, 2014). Para la obtención de los niveles de Inteligencia Múltiple se suministró un cuestionario llamado "Test de Inteligencias Múltiples" adaptación de Garden. Para la obtención del rendimiento académico, se procedió aq la recopilación de las notas bimestrales promediando dichas notas, extraídas de las fichas de registro de calificaciones del docente y evaluando su nivel según MINEDU (2016).

Procesamiento y Análisis de la Información. La estrategia utilizada fue la técnica estadística descriptiva; los datos fueron procesados mediante el paquete microsoft office Excel, el software estadístico SPSS versión 23.0 y el paquete estadístico de lenguaje de programación R- Proyect en su versión libre; se consideró un nivel de significancia de $P<0.05$. Para el análisis de datos y contraste de hipótesis se aplicó la estadística inferencial.

\section{Resultados}

Tabla 1.

Sexo De Los Estudiantes

\begin{tabular}{cc|c|c|c|} 
Niña & Frecuencia & Porcentaje & Porcentaje válido & Porcentaje acumulado \\
\hline Niño & 59 & 51,3 & 51,3 & 51,3 \\
Total & 115 & 48,7 & 48,7 & 100,0 \\
\multicolumn{4}{c}{ Fuente: Registro De Calificaciones Del Docente. }
\end{tabular}

Observamos en los resultados, la distribución de los estudiantes de las cuatro Instituciones Educativas privadas de Trujillo 131 según su sexo, donde las niñas tienen una mayor frecuencia estudiantil representando al $51.3 \%$ de los estudiantes y los estudiantes niños representan solo el $48.7 \%$. 
Tabla 2.

Rendimiento Académico De Los Estudiantes

\begin{tabular}{|c|c|c|c|c|c|c|c|c|}
\hline & Matemálicas & Comunicación & |ngles & $\begin{array}{l}\text { Artey } \\
\text { Cullura }\end{array}$ & $\begin{array}{l}\text { Educación } \\
\text { Fisica }\end{array}$ & $\begin{array}{l}\text { Personal } \\
\text { Social }\end{array}$ & Religión & $\begin{array}{l}\text { Ciencia y } \\
\text { Tecrologia }\end{array}$ \\
\hline $0-10$ & 17 & 7 & 0 & 1 & 0 & 1 & 0 & 8 \\
\hline $11-13$ & 65 & 45 & 53 & 61 & 48 & 41 & 30 & 71 \\
\hline $14-17$ & 33 & 59 & 61 & 53 & 67 & 68 & 85 & 36 \\
\hline $18-20$ & 0 & 4 & 1 & 0 & 0 & 5 & 0 & 0 \\
\hline & 115 & 115 & 115 & 115 & 115 & 115 & 115 & 115 \\
\hline
\end{tabular}

Fuente: Registro De Calificaciones Del Docente.

En la tabla 2 se muestran las ocho asignaturas evaluadas en la investigación, donde la asignatura que obtuvo mayor puntaje fue Personal Social, mientras que Matemáticas obtuvo los más bajos puntajes, también se encontró que el $15 \%$ de los estudiantes obtuvieron puntajes inferiores a 10 por lo que fueron calificados con $C$ es decir su logro de los aprendizajes esta en Inicio, mientras que en la asignatura de Personal Social el $4 \%$ de los estudiantes lograron un calificativo AD (logro destacado)

Tabla 3.

Rendimiento Académico y el Sexo De Los Estudiantes.

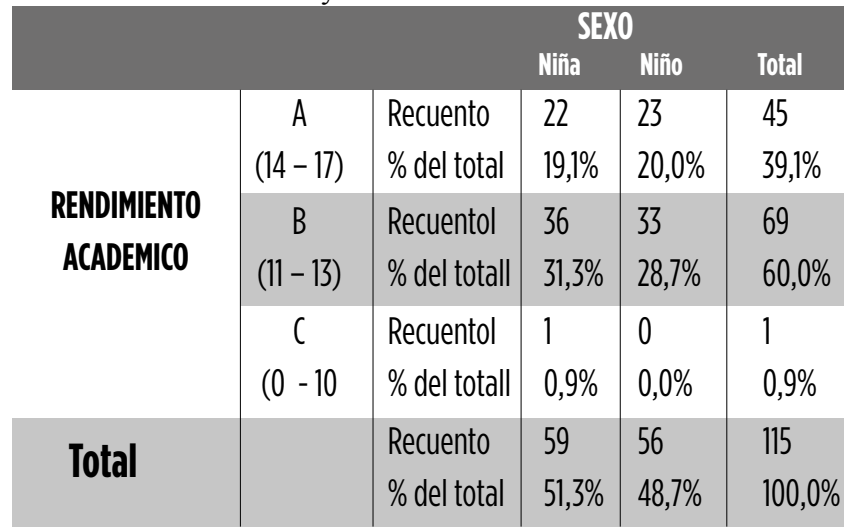

Fuente: Registro De Calificaciones Del Docente. $(t=0.088 ; p=0.47)$

Podemos observar el rendimiento académico promedio logrado por los estudiantes y su sexo, donde el puntaje logrado con mayor frecuencia logrado entre los estudiantes fue de 11 a 13 puntos, es decir que el 60\% de los estudiantes está en proceso de lograr los aprendizajes programados, y de ellos 33 son niños y 36 son niñas, mientras que el $39.1 \%$ de los estudiantes lograron un aprendizaje deseado para su nivel y de ellos 23 son niños y 22 son niñas, por ultimo solo una niña está en el inicio del aprendizaje deseado. Se aprecia también que no existe evidencia suficiente para determinar que exista diferencia entre el promedio del rendimiento académico según el sexo.

Tabla 4.

Inteligencia Múltiple De Los Estudiantes según su Sexo.

\begin{tabular}{l|c|c|c|c|c|c|c|c} 
& LING. & MAI. & ESP. & CINEST.A & MUS.L & INTER & INTRAP. & NAT \\
\hline General & 61,1 & 62,9 & 73,9 & 71,4 & 72,4 & 75,1 & 73,1 & 73,9 \\
Niños & 60,5 & 63,5 & 72,7 & 71,3 & 72,3 & 74,8 & 72,9 & 73,8 \\
\hline Niñas & 61,6 & 62,5 & 74,4 & 71,5 & 72,4 & 75,2 & 73,5 & 74,1
\end{tabular}

Fuente: Resultados de evaluación de Inteligencias Múltiples

En la tabla 4, observamos las ocho inteligencias de los estudiantes, siendo la Interpersonal la más desarrollada logrando un puntaje general entre los estudiantes de 75 pts., en esta inteligencia las niñas calificaron con 75.2 pts. y los niños con 74.8 , seguido por las inteligencias Naturalista y Espacial los cuales calificaron con 74 pts. ambos, la de menor desarrollo fue la Inteligencia Lingüística que logro en promedio 61 pts entre todos los estudiantes, de los cuales los niños calificaron con 60,5 pts. mientras que las niñas calificaron con mejor puntaje de 61.6 pts., entre tanto la inteligencia matemática en promedio califico con 63 pts. siendo los niños con mayor desarrollo y las niñas con un puntaje poco menor (63.5 y 62.5 pts. respectivamente).

Tabla 5.

Correlación de las Inteligencias Múltiples y el Rendimiento Académico.

\begin{tabular}{|c|c|c|c|c|c|}
\hline \multirow[b]{2}{*}{ 응 흘 } & \multirow[b]{2}{*}{$\begin{array}{l}\text { Inteligencia } \\
\text { LINGǗsTICA } \\
\text { Inteligencia } \\
\text { MATEMATICA }\end{array}$} & \multirow[b]{2}{*}{$\begin{array}{l}\text { Coeficiente de } \\
\text { correlación } \\
\text { Sig. (bilateral) } \\
\text { Coeficiente de } \\
\text { correlación } \\
\text { Sig. (bilateral) }\end{array}$} & \multirow{2}{*}{$\begin{array}{c}\begin{array}{c}\text { Matemática, } \\
\text { ceencia y } \\
\text { Tecnología }\end{array} \\
, 191^{*} \\
, 041 \\
, 363^{* *} \\
, 000\end{array}$} & \multirow{2}{*}{$\begin{array}{c}\begin{array}{c}\text { Inglés, Ante, } \\
\text { Educticion Fisica } \\
\text { y Religión }\end{array} \\
\text {,026 } \\
, 782 \\
, 280^{* *} \\
, 002\end{array}$} & \multirow{2}{*}{\begin{tabular}{|c}
$\begin{array}{c}\text { Comunicación } \\
\text { Y Peersonal } \\
\text { Social }\end{array}$ \\
, $483^{* *}$ \\
, 000 \\
,- 049 \\
, 605
\end{tabular}} \\
\hline & & & & & \\
\hline & $\begin{array}{l}\text { Inteligencia } \\
\text { ESPACIAL }\end{array}$ & $\begin{array}{l}\text { Coeficiente de } \\
\text { correlación } \\
\text { Sig. (bilateral) }\end{array}$ & $\begin{array}{l}, 311^{* *} \\
, 001\end{array}$ & $\begin{array}{l}, 468^{* *} \\
, 000\end{array}$ & $\begin{array}{l}-, 056 \\
, 554\end{array}$ \\
\hline & $\begin{array}{l}\text { Inteligencia } \\
\text { CINESTESICA }\end{array}$ & $\begin{array}{l}\text { Coeficiente de } \\
\text { correlación } \\
\text { Sig. (bilateral) }\end{array}$ & $\begin{array}{l}, 507^{* *} \\
, 000\end{array}$ & $\begin{array}{l}, 719^{* *} \\
, 000\end{array}$ & $\begin{array}{l}191^{*} \\
, 041\end{array}$ \\
\hline & $\begin{array}{l}\text { Inteligencia } \\
\text { MUSICAL }\end{array}$ & $\begin{array}{l}\text { Coeficiente de } \\
\text { correlación } \\
\text { Sig. (bilateral) }\end{array}$ & $\begin{array}{l}185^{*} \\
, 047\end{array}$ & $\begin{array}{l}, 366^{* *} \\
, 000\end{array}$ & $\begin{array}{l}, 015 \\
, 875\end{array}$ \\
\hline & $\begin{array}{l}\text { Inteligencia } \\
\text { INTERPERSONAL }\end{array}$ & $\begin{array}{l}\text { Coeficiente de } \\
\text { correlación } \\
\text { Sig. (bilateral) }\end{array}$ & $\begin{array}{l}, 241^{* *} \\
, 009\end{array}$ & $\begin{array}{l}103 \\
, 273\end{array}$ & $\begin{array}{l}, 580^{* *} \\
, 000\end{array}$ \\
\hline & $\begin{array}{l}\text { Inteligencia } \\
\text { INTRAPERSONAL }\end{array}$ & $\begin{array}{l}\text { Coeficiente de } \\
\text { correlación } \\
\text { Sig. (bilateral) }\end{array}$ & $\begin{array}{l}, 220^{*} \\
, 018\end{array}$ & $\begin{array}{l}, 210^{*} \\
, 024\end{array}$ & $\begin{array}{l}, 002 \\
, 985\end{array}$ \\
\hline & $\begin{array}{l}\text { Inteligencia } \\
\text { NATURALISTA }\end{array}$ & $\begin{array}{l}\text { Coeficiente de } \\
\text { correlación } \\
\text { Sig. (bilateral) }\end{array}$ & $\begin{array}{l}, 407^{* *} \\
, 000\end{array}$ & $\begin{array}{l}, 348^{* *} \\
, 000\end{array}$ & $\begin{array}{l}184^{*} \\
, 049\end{array}$ \\
\hline
\end{tabular}

* La correlación es significativa en el nivel 0,05 (2 colas). **. La correlación es significativa en el nivel 0,01 (2 colas). 
Estos datos demuestran que aquellos alumnos que tenían una alta performance en las asignaturas de Comunicación y Personal Social (segundo factor) correlacionaban directamente con las inteligencias lingüística, interpersonal y en baja correlación con la inteligencia cinestésica y la inteligencia Naturalista, en general registraban muy buenas habilidades verbales y poseían un talento para entender, comprender a los demás y comunicarse con ellos, gracias a ello el estudiante tiene facilidad para hablar y relacionarse con sus compañeros, sacando provecho en las asignaturas respectivas.

En la misma línea, aquellos que tenían alto rendimiento en las disciplinas de Matemática y Ciencia y Tecnología. (tercer factor), correlacionaban con las Inteligencias Matemáticas, Espacial, Cinestésica y Naturalista, eran aquellos que tenían talento para razonar y pensar lógicamente utilizando la observación y deducción lógica sin recurrir a símbolos verbales tienen la capacidad de decisión, la ética personal, la integridad, la empatía, el altruismo. Se reconoce en el alumno que prefiere y sabe trabajar solo, puede reflexionar sobre sus propias acciones, percibiendo las cosas visualmente, y con habilidad de formar modelos mentales en tres dimensiones. Todas estas cualidades juntas de las inteligencias son desarrolladas por los ingenieros, arquitectos

Por otro lado, aquellos estudiantes que registraban alto rendimiento en Ingles, Arte, Educación Física y Religión (primer factor) correlacionaban con las inteligencias Matemática, Espacial, Cenestésica, Musical y Naturalista mostraban habilidades relacionadas con el control y desarrollo del movimiento corporal, la resolución de problemas lógicas y visual espacial, la percepción de sonidosy la producción musical, talento para comprender sus sentimientos y efectuar discriminaciones finas entre sus emociones con una capacidad para aplicar estas introspecciones a la conducta efectiva (Fernández, 2016).

\section{Discusión}

En el ámbito internacional Pérez (2015), en su trabajo que tuvo como objetivo definir las múltiples inteligencias más desarrolladas por estudiantes con bajo rendimiento en Matemática, encontró que los estudiantes no tienen desarrolladas de forma equilibrada las inteligencias múltiples y que presentan en manera más predominante las inteligencias de tipo musical/rítmica. La inteligencia interpersonal está desarrollada en el $56.25 \%$ de los estudiantes, la intrapersonal en 56.25\%, Musical 59.38\%, Kinestésico 15.63\%, Espacial 6.25\%, Matemática $15.63 \%$ y Lingüística en $25 \%$. Se concluye que la mayoría de los estudiantes que presentan bajo rendimiento en Matemática no tienen desarrollada la inteligencia lógico matemática. Resultados semejantes a los encontrados en la presente investigación, puesto que también se encontró correlación entre la inteligencia lógico matemática y la asignatura de Matemática, a su vez que dichos alumnos no tenían desarrollada la inteligencia en niveles altos, incluso se encontró un nivel medio en el 17\% de la muestra.

En el ámbito nacional Aliaga et. al (2012) encontró que la inteligencia lógico-matemática se correlaciona más elevadamente con el rendimiento en matemáticas que con el rendimiento escolar general, así mismo, se comprueba que esta inteligencia y secundariamente la inteligencia lingüística se relacionan con el rendimiento general; $y$ que en el rendimiento en matemáticas además de la inteligencia lógico-matemática también juegan un rol pero menor las inteligencias cenestésica, musical, intrapersonal, lingüística y espacial, por lo cual se concluyó que las inteligencias múltiples si se relacionan significativamente. Se concuerda con estos resultados debido a que son semejantes a los ya expuestos, dando mayor credibilidad a lo importante que es desarrollar las inteligencias múltiples en el ámbito educativo, ya que se correlacionan sig- 
nificativamente las inteligencias múltiples y el rendimiento académico.

\section{Conclusiones}

1. En función de los resultados, se concluye que existe correlación significativa entre las inteligencias múltiples y el rendimiento académico en los estudiantes de las Instituciones Educativas privadas del sector urbano de Trujillo. Asegurando que los jóvenes estudiados exhiben mejor rendimiento en aquellas áreas en las que se consideran más capaces.

2. El $60 \%$ de los estudiantes lograron un aprendizaje previsto del rendimiento académico, siendo la asignatura Personal Social con un logro previsto de mayor puntaje (14.4), seguido por Religión con logro previsto (14,2 ptos) y en último lugar la asignatura Matemática estando en inicio del aprendizaje con un promedio global de 12,4 puntos.

3. La inteligencia múltiple más desarrollada es la inteligencia Interpersonal con 75 en general entre los estudiantes, seguido por la inteligencia, espacial y naturalista ambos con 74 ptos, por otro lado las inteligencias de menor puntuación fueron las inteligencias lingüísticas con 61ptos y la inteligencia matemática que obtuvo 63 ptos.

4. Se determinó que las niñas lograron mayor puntaje en las asignaturas de matemáticas, educación física, religión y Ciencia y Tecnología, mientras que los niños lograron un mejor puntaje en Comunicación, Ingles y Personal Social, ante lo expuesto se concluyó que no existe evidencia suficiente para determinar diferencia entre el promedio de notas del rendimiento

académico según el sexo de los alumnos.

5. Por último, la inteligencia Interpersonal es la de mayor desarrollo entre los alumnos, donde

las niñas calificaron con 75.2 pts. y los niños con
74.8, la Inteligencia Lingüística se determinó como la inteligencia de menor desarrollo entre todos los estudiantes, de los cuales los niños calificaron con 60,5 pts. y las niñas con 61.6 pts., seguido por la inteligencia matemática, donde los niños obtuvieron 63.5 y las niñas con 62.5 pts., por lo cual no existe evidencia suficiente para concluir en una diferencia entre las inteligencias múltiples y el sexo del alumno. 


\section{Referencias}

Aliaga J, Ponce C., Bulnes M., Elizalde R., Montgomery W., Gutiérrez V., Delgado E., Perea J, Torchiani R. (2012). "Las Inteligencias Múltiples: Evaluación Y Relación Con El Rendimiento En Matemática En Estudiantes Del $5^{\circ}$ De Secundaria De Lima" UNMSM. Lima: IIPSI (15). pp 163 - 202

Antunes, C. (2005) Las inteligencias múltiples. Cómo estimularlas y desarrollarlas. Lima: Edit. Narcea y Alfaomega

Barraza R. y Gonzales M., (2016). Rendimiento Académico y Autopercepción de Inteligencias Múltiples e Inteligencia Emocional en Universitarios de Primera Generación. Revista Electrónica "Actualidades Investigativas en Educación" (vol16. ppl-23) DOI: http://dx.doi.org/10.15517/aie.v16i2.23930

Castillo C. (2013) Estudio De Las Inteligencia Múltiples y de las Pautas Educativas en Alumnos con un Marcada Fracaso Escolar. Universidad Internacional de La Rioja Máster Universitario en Neuropsicología y Educación. La Rioja-España

Fernández, A. (2016). Una reflexión teórico-práctica sobre la educación hoy. Pensamiento Americano, 9(17), 105-120.

Flores N. (2012). Influencia de la Inteligencia General y Emocional, y de la Capacidad de Relación Sobre el Rendimiento Académico. Universidad Internacional de La Rioja. La Rioja-España.

Flores, M. (2010) Aplicación de estrategias innovadoras en base a las inteligencias múltiples para promover aprendizajes significativos en niños de 4 años de edad de la institución educativa Villa María de la ciudad de Tacna. Universidad Privada de Tacna. Perú

Gardner (2011). Inteligencias Múltiples: La Teoría En La Práctica. Biblioteca Howard Gardner Ed. Paidos Ibérica

Gardner, H. (1983). Frames of Mind: The Theory of Multiple Intelligences. Universidad de Michigan. Ed. Basic Book. Estados Unidos

Hernández, Fernández y Baptista, (2014). Metodología de la Investigación. (6ed.). México: McGraw-Hill Interamericana.

Higuera Ojito, V. (2013). Análisis económico de la demanda educativa en programas de Ciencias Económicas: un modelo de elección basado en un logit binomial. Ad-Gnosis, 2(2), 13-21
MINEDU (2016) Fascículo De Gestión Escolar Centrada En Los Aprendizajes. Recuperado de: wnw.minedu. gob.pe/n/xtras/fasciculo_gestion_escolar_ centrada_ en_aprendizajes.pdf

Pérez S.J. (2015). Inteligencias Múltiples más Desarrolladas en Estudiantes de Tercero Básico con Bajo Rendimiento en Matemática en un Colegio Privado Ubicado en el Municipio de San José Pinula en el Departamento de Guatemala. Universidad de San Carlos de Guatemala. Guatemala.

Scheaffer R., Mendenhall W. y Ott L. (2007) Elementos de muestreo. ( $6^{\circ}$ ed.). Madrid, España: Editorial Paraninfo. 